\title{
ORDER-THEORETICAL CONNECTIVITY
}

\author{
T.A. RICHMOND \\ Department of Mathematics \\ Western Kentucky University \\ Bowling Green KY 42101, U. S. A. \\ and \\ R. VAINIO \\ Department of Mathematics \\ Åbo Akademi \\ SF-20500 Åbo, Finland \\ (Received December 29, 1989 and in revised form February 10, 1990)
}

\begin{abstract}
Order-theoretically connected posets are introduced and applied to create the notion of $T$-connectivity in ordered topological spaces. As special cases $T$-connectivity contains classical connectivity, order-connectivity, and link-connectivity.
\end{abstract}

Key Words AND Phrases: Topologies on posets, links, maximal chains, order-theoretical connectivity, order-connectivity, link-connectivity, and T-connectivity.

AMS Subject Classification Codes: 54D05, 54 F05.

\section{INTRODUCTION}

In the literature on order and topology (ordered topological spaces, topological lattices, order-determined topologies, etc.) connectivity has generally been introduced as a topological property. Given some natural compatibility between topology and order, much is known about the impact different connectivity properties have on the order in question. Classical theory (in case of total order), as well as [2], [4], and [5], have revealed the close relationship between topological connectivity on one hand and the order-theoretical properties "conditionally complete" and "order dense" on the other. The aim of this note is to define a concept of order-theoretical connectivity (using conditional completeness and order density), and then to apply this concept to the theory of ordered topological spaces. First, some relevant remarks on (partial) order and connectivity will be given.

A triple $S \equiv(S, \tau, o)$ consisting of a set, an order $o$ and a topology $\tau$ on that set is called an ordered topological space. The space $S$ is called order-connected (cf. [1]) if all order-preserving continuous maps from $S$ to the space 2 are constants $(2 \equiv$ the set $\{0,1\}$ endowed with discrete topology and natural order); connected (path-connected) if it is connected (path-connected) as a topological space; and link-connected if any two points of $S$ can be joined by a finite set of connected links of $S$ (link between $x$ and $y$ $\equiv$ maximal chain of $[x, y])$. A class $\mathcal{C}$ of subsets of a given set $S$ is called a connectivity system (cf. [2]) if any set $A \subseteq S$ is a member of $\mathcal{C}$ whenever for all $x, y \in A$ there are sets $C_{1}, C_{2}, \ldots, C_{n} \in \mathcal{C}$ in $A$ with $x \in C_{1}, y \in C_{n}$, and $C_{i} \cap C_{i+1} \neq \emptyset$ for $i=1,2, \ldots, n-1$. The classes of order-connected, or connected, or path-connected, or link-connected sets constitute connectivity systems. 
An ordered topological space is a $T_{i}$-ordered space (an $i$-space) if every maximal chain inherits a topology which is finer than or equal to (which coincides with) its own interval topology (see [2], [5]). Note that for any lattice all topologies between the interval topology and Birkhoff's order topology are i-topologies (cf. [5]).

"Conditionally complete" and "order dense" will be abbreviated "cc" and "od", respectively. A subchain of an ordered set is called cc (od) if it is cc (od) regarded as an ordered space in its own right. It is important to recall that

(A) a chain is connected in its interval topology iff it is od and cc;

(B) an ordered set is od (cc) iff all maximal chains of it are od (cc);

(C) an od and cc chain between two points of a given ordered set is always a complete link.

\section{ORDER-THEORETICAL CONNECTIVITY}

Let $P$ be an arbitrary poset. Regard the equivalence relation $\sim^{\text {ord }}$ defined in $P$ by $a \sim$ ord $b \Longleftrightarrow a$ and $b$ are joined by a finite set of od and cc links in $P$.

The $\sim$ ord classes are called the order-theoretical components in $P$. In case of only one equivalence class, $P$ is order-theoretically connected. The order-theoretically connected subsets (defined in the obvious way) of any poset are a connectivity system in the sense of [2]. Moreover, a poset is order-theoretically connected iff it is link-connected in its interval topology (or equivalently, in any of its i-structures).

Proposition 1. For any $a \in P$, the order-theoretical component of a equals the maximal order-theoretically connected subset of $P$ containing $a$.

Lemma 2. In any product poset $P \equiv \Pi P_{i}$, all projections of an od and cc link $J$ are od and cc links.

Proof. For a topological proof, endow $P$ and the $P_{i}$ 's with Birkhoff's order topology $o$, note that $J$ is a connected set in $o(P)$ and that all projections are continuous maps $o(P) \rightarrow o\left(P_{i}\right)$. Thus $p r_{i}(J)$ is a connected chain in $o\left(P_{i}\right)$, and since $p r_{i}(J)$ carries a structure which is finer than its own interval topology it is an od and cc chain.

Proposition 3. The product poset $P \equiv P_{1} \times P_{2} \times \ldots \times P_{n}$ is order-theoretically connected iff every factor is. The order-theoretical component of $a \equiv\left(a_{1}, a_{2}, \ldots, a_{n}\right) \in P$ equals the product of the order-theoretical components of the $a_{k}$ 's $(k=1,2, \ldots, n)$.

Proof. To see the first part for $n=2$, take $a_{2} \in P_{2}$, express the product set $P_{1} \times P_{2}$ as $\bigcup_{a_{1} \in P_{1}}\left[\left(P_{1} \times\left\{a_{2}\right\}\right) \cup\left(\left\{a_{1}\right\} \times P_{2}\right)\right]$, and use Lemma 2 . Then proceed via induction over the natural numbers. The remaining part is straightforward.

EXAMPle 4 (denumerable products). Consider the Euclidean plane, let $l_{k}$ denote the line segment $\left(y=\frac{1}{k}, 0 \leq x \leq 1\right)$ and $m_{k}$ the line segment connecting the right endpoint of $l_{k}$ and the left endpoint of $l_{k+1}$. Define the lattice $L$ to be the union of all the $l_{k}$ 's and $m_{k}$ 's $\left(k \in \mathbf{Z}_{+}\right.$) endowed with the natural order of the plane. Obviously, $L$ is order-theoretically connected. To see that the product lattice $P \equiv L^{\mathbf{Z}_{+}}$is not, take pairs of points $a_{n}, b_{n}$ in $L$ which can be joined by a set of no less than $n$ od and cc links of $L$ and note $\left(a_{n}\right) \sim\left(b_{n}\right)$ is not satisfied in $P$ (use Lemma 2).

EXAMPLE 5 (basic remarks). The od and cc lattices form a class of order-theoretically connected structures which is closed under formation of arbitrary products. A lattice is od and cc iff all maximal chains (or all links) are order-theoretically connected. As was demonstrated in Lemma 2, projections preserve order-theoretical connectivity. However, 
a lattice with prime ideals (for instance any distributive lattice) allows a lattice-morphism onto the lattice 2. This shows that order-theoretical connectivity cannot be characterized via morphisms (and, of course, that morphisms do not preserve order-theoretical connectivity). Cantor's teepee (cf. [3]) with base interval $[0,1]$ and top $(1,1)$ is a connected subset of $\mathbf{R}^{2}$; nevertheless, all its order-theoretical components are singletons (which is a quite natural order-theoretical property of this structure). Finally, note that the topological closure of the lattice $L$ of Example 4 (the Euclidean topology) consists of two order-theoretical components, although $L$ is order-theoretically connected.

In ordered topological spaces $S$, order-theoretical connectedness relates to pathconnectedness, as demonstrated below.

Proposition 6. Suppose each $x \in S$ has an order-theoretically connected nerghborhood. Then $S$ is order-theoretically connected if $S$ is path-connected.

Proof: Suppose $x, y \in S$ and $p: \mathbf{I} \rightarrow S$ is a path between $x$ and $y$. For each $z \in p(\mathbf{I})$, let $N_{z}$ be an order-theoretically connected neighborhood of $z$, and let $M_{z} \subseteq N_{z}$ be an open neighborhood of $z$. Consider $\left\{N_{i}\right\}_{i=1}^{n}$ where $\left\{M_{i}\right\}_{i=1}^{n}$ is a subcover of $p(\mathrm{I})$ from $\left\{M_{z}: z \in p(\mathrm{I})\right\}$. Now $\left(\cup_{i=1}^{k} N_{i}\right) \cap\left(\cup_{i=k+1}^{n} N_{i}\right) \neq \emptyset$ for any $1 \leq k \leq n$, for otherwise $p^{-1}\left(\cup_{i=1}^{k} M_{i}\right) \cup p^{-1}\left(\cup_{i=k+1}^{n} M_{i}\right)$ would separate $I$ for some $k$. Since the order-theoretically connected subsets of $S$ form a connectivity system, it follows that $x$ and $y$ are in the same order-theoretical component and thus $S$ is order-theoretically connected.

An obvious sufficient condition for order-theoretical connectedness to imply pathconnectedness is that every cc and od link from $x$ to $y$ be a path from $x$ to $y$. Applying this and the proposition we finally get

COROllary 7. An open subset of $\mathbf{R}^{n}$ is path-connected iff it is order-theoretically connected, given the usual topology and order on $\mathbf{R}^{n}$.

\section{T-CONNECTIVITY}

Order-theoretical connectedness is now employed to create a natural connectivity concept in the category of (partially) ordered topological spaces and order-preserving continuous maps (here called morphisms).

Let $T$ denote an ordered topological space.

The ordered topological space $S$ is called $T$-connected if for every morphism $\varphi: S \rightarrow T$ the image $\varphi(S)$ is an order-theoretically connected set in $T$. A subset of an arbitrary ordered topological space is a $T$-connected set if it is a $T$-connected space (regarded as a subspace). Every ordered topological space can be partitioned into maximal $T$-connected sets, the $T$-components of the space.

Obviously, the $T$-connected sets in any ordered topological space form a connectivity system in the sense of [2]. $T$-connectivity provides the realm for several well-known connectivity concepts. For instance, an ordered topological space is order-connected iff it is 2-connected. As a special case of this, note that a topological space is connected in the classical sense iff it is a 2-connected ordered topological space when equipped with the discrete order. A connected space endowed with any order is always an $\mathbf{R}$-connected ordered topological space. 
Proposition 8. Let $S$ be a $T_{i}$-ordered topological space. Consider the following statements

(1) $S$ is link-connected;

(2) $S$ is $T$-connected for all $T_{i}$-ordered topological spaces $T$;

(3) $S$ is $S$-connected;

(4) $S$ is order-theoretically connected.

Implications $(1) \Rightarrow(2) \Rightarrow(3) \Rightarrow(4)$ are always true. If $S$ is an $i$-space, then (4) $\Rightarrow(1)$.

Proposition 9. Everything stated in Proposition 3 holds for T-connectivity; moreover, $T$-connectivity is preserved by morphisms (the choice of $T$ is arbitrary, throughout).

Example 10 (denumerable products). The cc lattice $L$ of Example 4 endowed with Euclidean topology is $T$-connected for all $T_{i}$-ordered topological spaces $T$ (cf. Proposition 8). The product lattice $P \equiv L^{\mathbf{z}_{+}}$endowed with product topology is an i-space. Since the space $P$ cannot be link-connected (pick elements $\left(a_{n}\right),\left(b_{n}\right) \in P$ as in Example 4), Proposition 8 guarantees the existence of at least one $T_{i}$-ordered space $T$, for which $P$ is not $T$-connected.

EXAMPLE 11 (basic remarks). The set $L$ of the previous example is an $\mathbf{R}^{2}$-connected set in the Euclidean plane. However, the closure of $L$ (denoted by $\bar{L}$ ) consists of two $\mathbf{R}^{2}$-components, one of which fails to be a closed set in the space $\bar{L}$. Also, note that any $T_{3,5}$ topological space endowed with discrete order is totally $\mathbf{R}^{2}$-disconnected (i.e. all $\mathbf{R}^{2}$-components consist of only one point).

Again let $T$ be any ordered topological space.

Proposition 12. A connected ordered topological space $S$, in which every point has a $T$-connected neighborhood, is $T$-connected.

Proof. For $x, y \in S$, let $x \sim^{T} y$ denote that for all morphisms $\varphi: S \rightarrow T$ the points $\varphi(x), \varphi(y)$ are joined by a finite set of od and cc links in $\varphi(S)$. Take $a \in S$, define the subset $A$ of $S$ by $x \in A \Longleftrightarrow x \sim^{T} a$, and prove $A=S$ by showing $A$ is open and closed as follows. First, take $b \in A$ and let $V$ be a $T$-connected neighborhood of $b$. For any $x \in V, x \sim^{T} b \sim^{T} a$, and thus $x \in A$. Then, for $b$ in the closure of $A$ and $V$ as above, $A \cap V \neq \emptyset$ and every $x \in A \cap V$ satisfies $b \sim^{T} x \sim^{T} a$, which implies $b \in A$. •

\section{REFERENCES}

1. D.C.J. BURGESS and S.D. MCCARTAN, Order-continuous functions and order-connected spaces, Proc. Camb. Phil. Soc. 68 (1970), pp 27-31.

2. M. ERnÉ and R. VAINIO, Connectivity in lattice-ordered spaces, to appear in Math. Nachr.

3. L.A. STEEN and J.A. SEEBACH, Counterexamples in topology, Holt - Rinehart - Winston, New York 1968.

4. R. VaInio, Connectedness properties of lattices, Canad. Math. Bull. 29 (1986), pp 314-320.

5. R. VAINIO, A maximal chain approach to topology and order, Internat. J. Math. and Math. Sci. 11 no. 3 (1988), pp 465-472. 


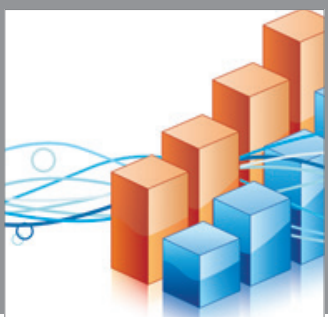

Advances in

Operations Research

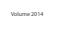

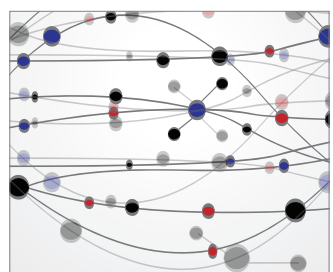

\section{The Scientific} World Journal
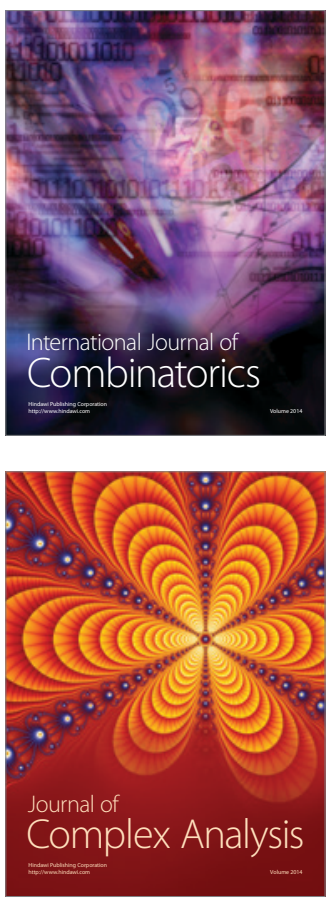

International Journal of

Mathematics and

Mathematical

Sciences
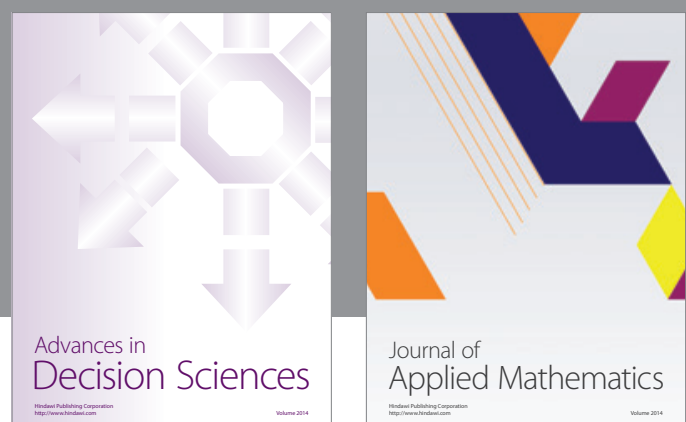

Journal of

Applied Mathematics
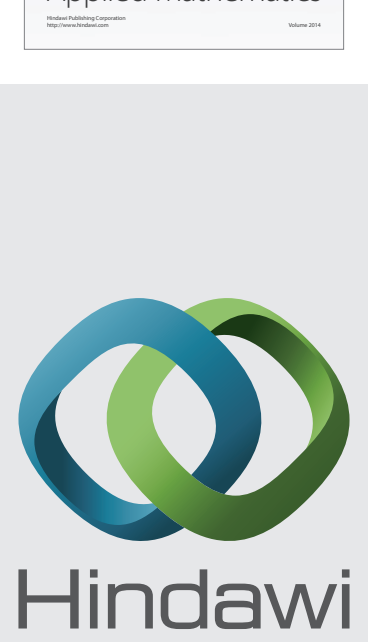

Submit your manuscripts at http://www.hindawi.com
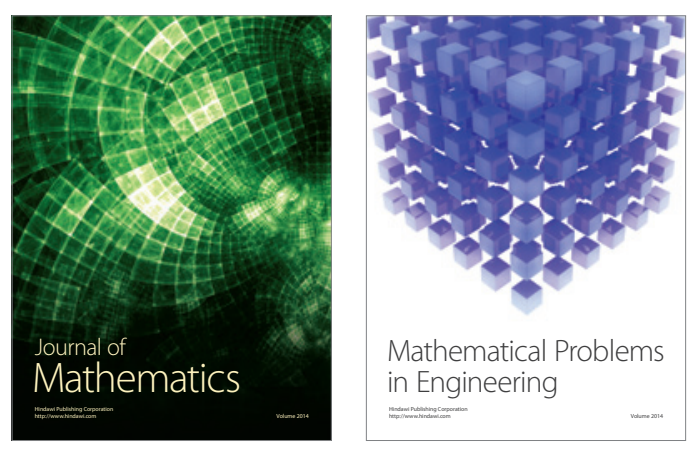

Mathematical Problems in Engineering
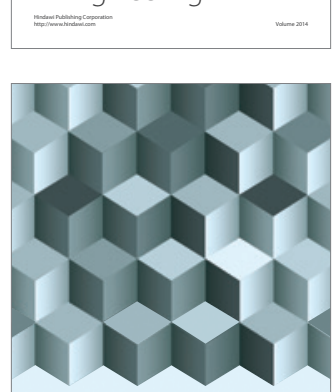

Journal of

Function Spaces
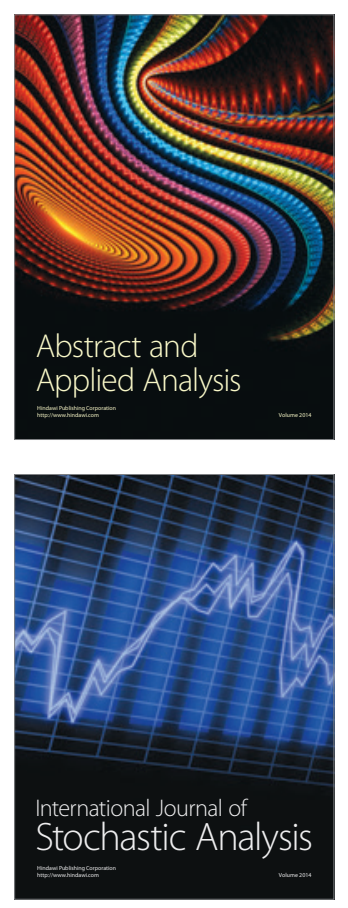

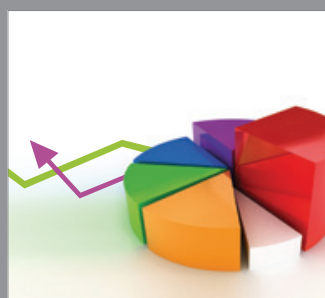

ournal of

Probability and Statistics

Promensencen
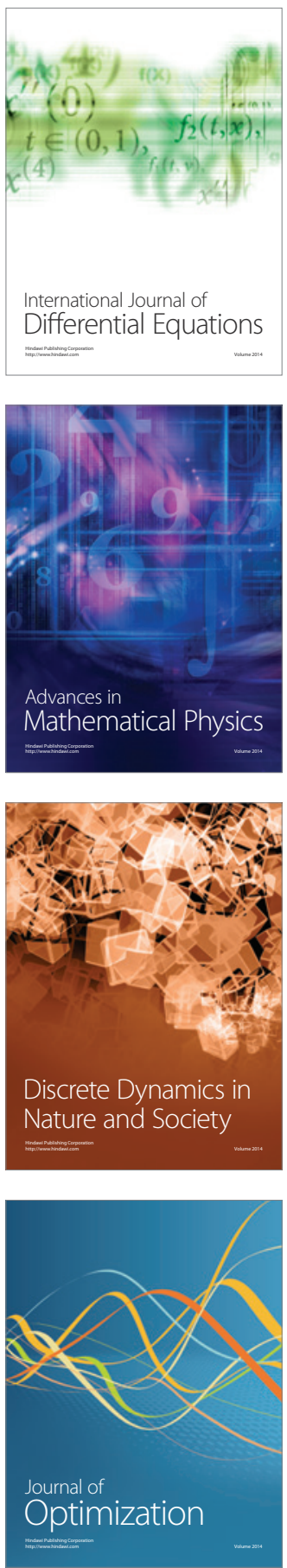